\title{
Plastic Surgery Techniques for the Improvement of Outcomes of Complex Obstetric Fistula Repairs
}

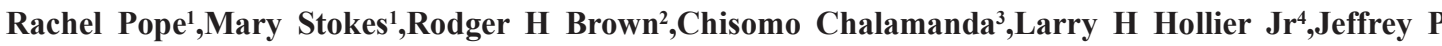 \\ Wilkinson ${ }^{1}$ \\ 'Division ofGlobalWomen'sHealth,DepartmentofObstetricsandGynecology,BaylorCollegeofMedicine, Houston, TX, USA \\ ${ }^{2}$ Rodger H Brown, Division of Plastic Surgery, Department of Surgery, Houston Methodist,Houston, TX, USA \\ ${ }^{3}$ Chisomo Chalamanda, Fistula Care Center, Lilongwe, Malawi \\ ${ }^{4}$ Larry H Hollier Jr, Division of Plastic Surgery, Department of Surgery, Baylor College ofMedicine, Houston, TX, USA
}

DOI: http://dx.doi.org/10.3126/njog.v13i2.21735

\begin{abstract}
Aims: As part of a larger study on the outcomes of obstetric fistula surgery, a review on patient outcomes when using gracilis muscle and/ or Singapore flaps was conducted.

Methods: The database queried includes over 1700 patients. Fifty-six cases were identified having had either a gracilis muscle and or a Singapore flap as part of the repair.

Results: Twenty-one patients had a Singapore flap only. Median age was 26 years (19-55), four had one prior repair and two had two prior repair attempts. Nine cases were Goh type 3 and nine were Goh type 4 indicating urethral involvement. $71 \%$ ( $n=15)$ were $>3$ centimeters in diameter. Median estimated blood loss (ebl) was $200 \mathrm{ml}$ and average OR time was 2.5 hours. Median catheter duration 17 days (13-25). $81 \%(\mathrm{n}=17)$ were dye test negative, with an average pad weight of 19.2 grams. 19 patients had a gracilis muscle flap alone with median age of 43 (23-70). Four had one previous repair, one had four previous repairs. Nine were Goh type 3 and eight were Goh type $4.70 \%$ ( $\mathrm{n}=12$ ) had a fistula $>3 \mathrm{~cm}$ in diameter and $88 \%(\mathrm{n}=15)$ had type iii considerations (previous repair attempt, circumferential, or severe scarring). Median ebl was $250 \mathrm{ml}$ and average operative time was two hours and 30 minutes. Median catheter duration was 17 days (14-31). 82\% $(n=14)$ had negative dye tests, with average pad weight of 19 grams. 16 patients had both a Singapore and a gracilis. Median age was 31 (15-70), nine were Goh type 3, seven were Goh type 4. 87.5\% $(\mathrm{n}=14)$ had a fistula that was more than $3 \mathrm{~cm}$ in diameter and $87.5 \%(\mathrm{n}=14)$ were type iii. Median ebl was $300 \mathrm{ml}(250-1000 \mathrm{ml})$, and average operative time was 3 hours and 45 minutes. $81 \%(\mathrm{n}=13)$ had a negative dye test, with two patients going home positive and returning negative over the course of four months. Average pad weight was 18.9 grams.
\end{abstract}

Conclusions: For large fistulas with a significant amount of vaginal tissue loss, the Singapore flap is a potential option for improved outcomes. For recurrent cases and those with poor quality tissue, the gracilis muscle may lead to overall improved outcomes. Overall, these techniques are useful for complex obstetric fistula cases where outcomes are generally less favorable. Further prospective studies are needed.

Keywords: gracilis flap, obstetric fistula, Singapore flap, vesico-vaginal fistula.

\section{INTRODUCTION}

Complex obstetric fistulas comprise approximately $5-15 \%$ of all vesico-vaginal and recto-vaginal fistulas resulting from obstructed labor. These cases are fraught with anatomical challenges such as severe scarring and vaginal stenosis, small bladder capacity, short or absent urethras, and previous repair attempts without success. Innovative techniques are needed to achieve healing in many of these cases. ${ }^{1,2}$ In a fresh-tissue lab, plastic surgeons demonstrate two techniques that could be replicated for women

\section{CORRESPONDENCE}

Dr Rachel Pope

Division of Global Women's Health, Department of Obstetrics and Gynecology, Baylor College of Medicine, One Baylor Plaza, 77030, Houston, TX, USA

Email: Rachel.pope@bcm.edu with complex obstetric fistulas. The first technique is the fasciocutaneous pudendal flap, known as the Singapore flap. ${ }^{3,4}$ The second is the gracilis muscle flap, commonly used in fistulas due to radiation therapy and in recto-vaginal fistulas. ${ }^{5}$ Both flaps maintain their vascular supply, thus improving tissue quality in a previously scarred vagina. Both flaps also can be performed with the same instruments already used for obstetric fistula and do not requiring stenting or additional equipment. Plastic surgeons traveled to the Fistula Care Center in Lilongwe, Malawi, to offer these techniques to women with complex fistulas and to simultaneously continue instructing obstetric fistula surgeons in performing the procedures.After two years of including these flaps as part of our repair techniques when appropriate, a review of our cases is warranted.Therefore, this is a retrospective review of surgeries performed at the Fistula Care Center in 
Lilongwe, Malawi including either a gracilis and/or Singapore flap as part of the repair technique.

\section{METHODS}

All participants consented for surgery and participation in the study which was part of a larger study on outcomes of obstetric fistula surgery. Ethical approval is granted by both the Malawian National Health and Sciences Research Committee and the Institutional Review Board of Baylor College of Medicine. All cases are entered into a RedCap database by research personnel and files are cleaned by clinical personnel. The database queried includes over 1700 patients. Fifty-six cases were identified having had either a gracilis muscle and or a Singapore flap as part of the repair.

\section{Singapore Flap}

After the vesico-vaginal and/or recto-vaginal fistula is repaired and vaginal scarring is released, there is often a defect of vaginal epithelium remaining. The Singapore flap serves as healthy tissue to cover this defect and reduce tension on the repair. This pudendal thigh flap is centered over the labial crural fold with its base at the perineal body. The blood supply from the posterior labial artery anastomoses with branches of the deep external pudendal artery, maintaining the viability of the tissue when the fascia is also kept intact. The flap is created with a scalpel and a diathermy pencil is used to raise and rotate it so that it may enter the vagina either through a tunnel or through a medio-lateral episiotomy.

\section{Gracilis Muscle Flap}

The gracilis muscle is identified two finger breadths posterior to the adductor longus and an incision is made. The muscle is dissected distally towards its insertion at the medial tibial condyle. It is detached at this tendinous insertion and brought back towards the vagina through dissection stopping at the vascular supply of the medial femoral circumflex artery. At this point it is tunneled through the vagina where it is sutured in place where needed.

If there is not adequate vaginal lining after placing the gracilis flap, often a Singapore flap is also needed. After four weeks of healing, participants use silicone vaginal dilators as needed to regain functional vaginal caliber.

\section{RESULTS}

Twenty-one patients had a Singapore flap only. Median age was 26 years (19-55), four had one prior repair and two had two prior repair attempts. Nine cases were Goh type 3 and nine were Goh type 4 indicating urethral involvement. $71 \%(n=15)$ were $>3$ centimeters in diameter. Median estimated blood loss (ebl) was $200 \mathrm{ml}$ and average OR time was 2.5 hours. Median catheter duration was 17 days (13-25). 81\% $(\mathrm{n}=17)$ were dye test negative, with an average pad weight of 19.2 grams.

Nineteen patients had a gracilis muscle flap alone with median age of 43 (23-70). Four had one previous repair, one had four previous repairs. Nine were Goh type 3 and eight were Goh type 4. 70\% (n=12) had a fistula $>3 \mathrm{~cm}$ in diameter and $88 \%(\mathrm{n}=15)$ had type iii considerations (previous repair attempt, circumferential, or severe scarring). Median ebl was $250 \mathrm{ml}$ and average operative time was two hours and 30 minutes. Median catheter duration was 17 days (14-31). 82\% ( $\mathrm{n}=14)$ had negative dye tests, with average pad weight of 19 grams.

Sixteen patients had both a Singapore and a gracilis. Median age was 31 (15-70), nine were Goh type 3, seven were Goh type 4. 87.5\% $(n=14)$ had a fistula that was more than $3 \mathrm{~cm}$ in diameter and $87.5 \%(n=14)$ were type iii. Median estimated blood loss was 300 $\mathrm{ml}(250-1000 \mathrm{ml})$, and average operative time was 3 hours and 45 minutes. $81 \%(n=13)$ had a negative dye test, with two patients going home positive and returning negative over the course of four months. Average pad weight was 18.9 grams.

\section{DISCUSSION}

Overall, we note a higher than expected success rate from these complex cases when using either the Singapore and/or gracilis flap. While criteria for the Singapore flap are a significant loss of vaginal tissue, criteria for the gracilis flap are less obvious. Criteria may include multiple previous failed repairs, and poor tissue quality, however, the latter being subjective. Therefore, appropriate case selection for each flap is to the discretion of the surgeon until more detailed indications are delineated.The only other published research outside of our center's on these flaps for complex obstetric fistula repairs have also demonstrated improved outcomes, likely due to restoration of original anatomy. ${ }^{6,7}$ 
Limitations of this study primarily center around its retrospective nature. It would be more useful to look prospectively at the use of each flap in a randomized controlled trial, however due to the heterogeneity of the injuries that constitute complex obstetric fistulas, this would be challenging. In addition, longer term follow-up would be useful.

\section{CONCLUSIONS}

Overall, the collaboration of plastic and gynecological surgeons led to success in improving repair techniques for this series of women with obstetric fistula. For large fistulas with a significant amount of vaginal tissue loss, the Singapore flap is a potential option for improved outcomes. For recurrent cases and those with poor quality tissue, the gracilis muscle may lead to overall improved outcomes. In cases with both defects, both flaps may be indicated. Overall, these techniques are useful for complex obstetric fistula cases where outcomes are generally less favorable. Further prospective studies are needed.

\section{ACKNOWLEDGEMENT}

The authors would like to thank Freedom from Fistula Foundation for the overall management and support of the Fistula Care Center.

\section{REFERENCES}

1. Pope R. Research in Obstetric Fistula Addressing Gaps and Unmet Needs. Obstetrics and Gynecology. 2018;131(5):86370 .

2. Pope R, Wilkinson J. Surgical innovation for obstetric fistula patients. BJOG. 2018.;125 (6):750.

3. Wee JTK and Joseph VT. A new technique of vaginal reconstruction using neurovascular pudendal-thigh flaps: a preliminary report. Plastic and Reconstructive Surgery. 1989;83 (4):701-9.

4. Pope RJ, Brown RH, Chipungu E, Hollier LH Jr, Wilkinson JP. The use of Singapore flaps for vaginal reconstruction in women with vaginal stenosis with obstetric fistula. BJOG. 2018;125(6):751-6.
5. Pope R, Brown RH, Chalamanda C, Hollier LH Jr, Wilkinson JP. The gracilis muscle flap for irreparable, "impossible," and recurrent obstetric fistulas. Int $\mathrm{J}$ Gynaecol Obstet. 2018;143(3):390-2

6. Browning A, Williams G, Petros P. Skin flap vaginal augmentation helps prevent and cure post obstetric fistula repair urine leakage: a critical anatomical analysis. BJOG. 2018;125(6):745-9.

7. Hamlin R, Nicholson C. Reconstruction of Urethra Totally Destroyed in Labour. BMJ. 1969;2:147-150 